\title{
Drain or not After Repair of Ventral Hernia with Mesh Ahmed Abd Elmawgoud. El-Tokhy
}

Department of General Surgery, Faculty of Medicine, Al-Azhar University, Egypt

Corresponding author: A. El-tokhy, Email.eltokhy81@ gmail.com phone.01153477679

\begin{abstract}
Background: Abdominal wall hernias are among the most common of all surgical problems. Ventral hernia repair (VHR) is a commonly performed operation, so hernia defect size affects operative time and surgical technique for repair of a ventral hernia. Incisional hernia is the most frequent postoperative complication following general surgery.
\end{abstract}

Objectives: This study was done to review and assess the difference between insertion of wound drain and the use of abdominal binder and percutaneous aspiration after open repair of ventral hernias and its effect on postoperative outcome.

Patients and methods: A total of 100 patients with non-complicated abdominal wall (ventral) hernia were enrolled to review and assess the difference between insertion of wound drain and the use of abdominal binder and percutaneous aspiration after open repair of ventral hernias and its effect on postoperative outcome. Patients were divided into two groups: Group (A) includes 50 patients with insertion of wound drain. Group (B) includes 50 patients with application of abdominal binder immediately postoperatively for 2 weeks.

Results: This study showed distribution of patients according to age, ranging from 27 years to 70 years with mean age of 49.2 years ( \pm 11.6 years). $25 \%$ of patients below $39,50 \%$ of patients below 50 and $75 \%$ of patients below 59.75. In our study, $60 \%$ of operations were carried out under spinal anesthesia and $40 \%$ of operations were carried out under general anesthesia. The hernial content of 100 patients was intestine in 28 cases, and omentum in 72 cases. There were developing of significant seroma in (30\%) of patients as follow: (17\%) at group A (with drain) and (13\%) at group B (without drain). Significant seroma was not seen in (70\%) of patients. Wound infection was developed in (18\%) of patients as follow; (12\%) for group A (with drain) and (6\%) for group B (without drain). Wound infection not seen in $(82 \%)$ of patients. As regard recurrence, $(2 \%)$ of total count only showing early recurrence that present at group B. Of total count only (9\%) complained of persistent postoperative pain as follows, 6 cases from group (A) and 3 cases from group (B).

Conclusion: It could be concluded that combined abdominal binder and percutaneous aspiration for selected cases were superior to insertion of wound drains as regards seroma formation, wound infection and postoperative return to normal activity with no significant difference as regards hematoma, postoperative pain, hospital stay and patient satisfaction.

Keywords: wound drain, abdominal binder, percutaneous aspiration, ventral hernia, polyprolene mesh.

\section{INTRODUCTION}

Ventral hernias are defined as a defect of the fascia in the anterior abdominal wall with or without a bulge. Clinical presentation varies from small incidental defects to giant and complicated hernias with fistulas and viscera located outside the abdominal cavity covered only by peritoneum and skin (loss of domain). The symptoms range from minor cosmetic concerns to severe pain and lifethreatening conditions such as bowel obstruction, incarceration, strangulation and perforation ${ }^{(\mathbf{1})}$.

Ventral hernia is one of the most common general surgical pathologies. An estimated 20 million patients with hernias are operated on worldwide every year, of which approximately $30 \%$ are ventral. An incisional hernia will develop in $10-15 \%$ of patients with an abdominal incision and the risk increases to up to $23 \%$ in those who develop surgical site infection. Incidence rates up to $69 \%$ have been reported in high-risk patients ${ }^{(2)}$.
Ventral hernia repairs are mostly elective $(90 \%)$ procedures, but the repair methods are highly variable. Repair methods are often not based on current best. practices and results can be disappointing. Few surgeons audit or record their outcomes outside of those countries with national registries ${ }^{(1)}$.

This study was conducted to review and assess the difference between insertion of wound drain and the use of abdominal binder and percutaneous aspiration after open repair of ventral hernias and its effect on postoperative outcome.

\section{PATIENTS AND METHODS}

This prospective controlled trial study included a total of 100 patients presented with non-complicated abdominal wall (ventral) hernia attending at the Surgical Department, Faculty of Medicine, Al-Azhar University Hospitals. Approval of the ethical committee and a written informed consent from all 
the subjects were obtained. This study was conducted between December 2016 to April 2017. Patients were divided into two groups: Group (A) includes 50 patients with insertion of wound drain. Group (B) includes 50 patients with application of abdominal binder immediately postoperatively for 2 weeks.

Inclusion criteria: All patients with ventral hernias with defect size between 2 and $12 \mathrm{~cm}$ in maximal dimension.

Exclusion criteria: Patients with infection, skin loss, large ventral hernias (drain is mandatory) and recurrent cases.

History taking: The following patient demographics and clinical information were obtained: age, gender, comorbidities, medical history (hypertension, diabetes, cardiac, COPD), surgical history, personal history of smoking or alcohol intake.

\section{Physical examination:}

Proper physical examination to confirm the diagnosis of ventral hernia, assess the presence of complication (e.g. obstruction, strangulation).

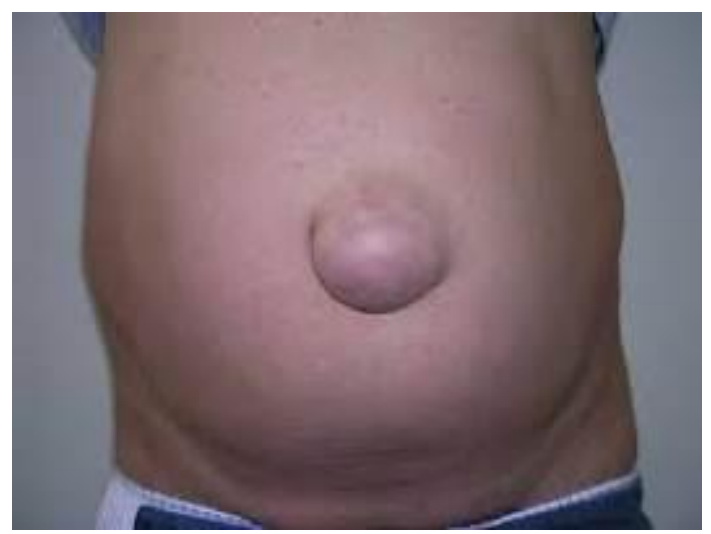

Figure (1): Ventral (umblical) hernia

\section{Preoperative preparation:}

Preoperative patient preparation included the correction of fluid and electrolytes imbalance, and the administration of antibiotic in the form of intravenous $3^{\text {rd }}$ generation cephalosporin preoperatively.

Routine preoperative laboratory investigations (CBC, Coagulation profile, liver and kidney function tests) to exclude ineligible cases.

Abdominal ultrasound was done to confirm the diagnosis of ventral hernia, to exclude the possibility of intra-abdominal cause of ventral hernia and to exclude the presence of any condition that needs to be operated upon in the same setting. ECG and Chest X-ray were done to assess the cardiopulmonary condition.

\section{Anesthesia:}

The decision regarding anesthetic options was left to the anesthetist in charge. The procedure was carried out using general anesthesia in 40 patients, intravenous midazolam or propofol infusion was administered by the anesthetist, as appropriate. The procedure was carried out using spinal anesthesia in 60 patients.

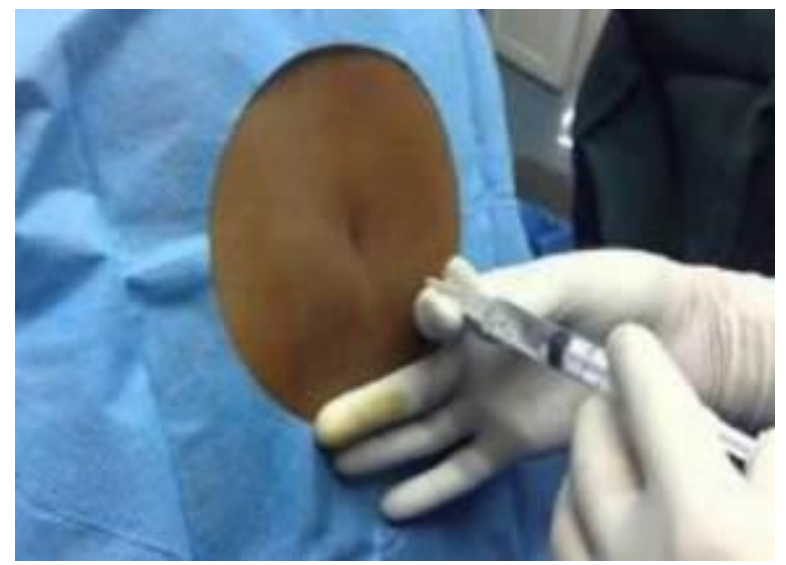

Figure (2): Spinal anesthesia

\section{Operative technique:}

Vertical or transverse incision was done. Meticulous dissection of the hernial sac until its neck proper was clearly identified. The aponeurotic fascia was cleared about $5 \mathrm{~cm}$ around the hernia defect. The fundus of the sac was meticulously dissected off the skin. Sac is opened and contents are dealt with according to the condition.

Defect size is measured either preoperatively (admission of fingers or ultrasound) or intraoperatively (sterilized tape).

Closure of the defect by approximation of the edges (herniorrhaphy) using non-absorbable 0 or 1 suture material, either transversally or vertically, whichever axis is shorter.

Application of polypropylene mesh that extends $5 \mathrm{~cm}$ all around the repair (either sutured by prolene sutures $2 / 0$ or mesh tucker).

Drainage of the subcutaneous dead space is indicated if the skin is widely undermined (skin flaps). The subcutaneous tissues are approximated, and skin closure is performed, sufficient for perfect hemostasis but non ischemic.

Application of abdominal binder immediately postoperatively for 2 weeks in half of patients.

Insertion of wound drains (suction drains) in the other half.

\section{Postoperative follow up:}

The patients were closely observed postoperatively for adequate pain control, urine output, blood gases, clinical examination and investigations were carried out regularly to follow up the patient general condition. 
Antibiotics were continued 3 days postoperatively in patients who recovered without complication. In patients who developed complication, the use of antibiotics was continued according to the patient's condition.

Anti-inflammatory agents were used for 14 days postoperatively.

The hernia repair was followed up to detect any complication as seroma, hematoma, and wound infection.

Follow up at outpatient clinic after 6 months for hernia recurrence.

Percutaneous aspiration of seroma in the follow up visits in the outpatient clinic after skin preparation by bovidone iodine (betadine).

Postoperative pain, patient satisfaction and return of normal activity were observed.

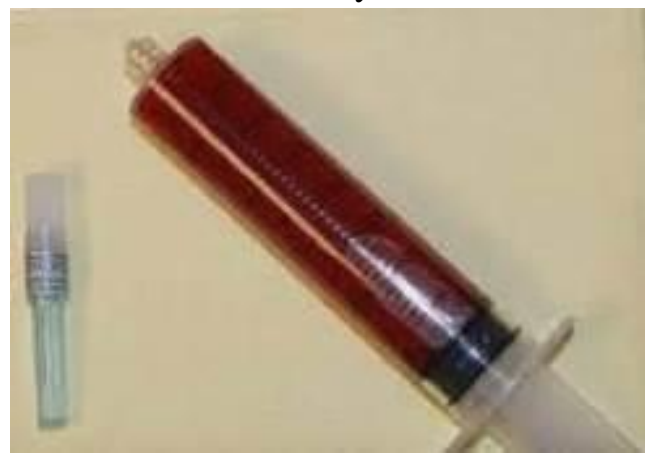

Figure (3)

\section{Statistical analysis}

Recorded data were analyzed using the statistical package for social sciences, version 20.0 (SPSS Inc., Chicago, Illinois, USA). Quantitative data were expressed as mean \pm standard deviation (SD). Qualitative data were expressed as frequency and percentage.

\section{The following tests were done:}

- Independent-samples t-test of significance was used when comparing between two means.

- Chi-square $\left(\mathrm{x}^{2}\right)$ test of significance was used in order to compare proportions between two qualitative parameters.

- The confidence interval was set to $95 \%$ and the margin of error accepted was set to $5 \%$. The p-value was considered significant as the following:

- Probability (P-value)

P-value $<0.05$ was considered significant.

$\mathrm{P}$-value $<0.001$ was considered as highly significant.

$\mathrm{P}$-value $>0.05$ was considered insignificant.

\section{RESULTS}

Table (1) shows distribution of patients according to age, ranging from 27 years to 70 years with mean age of 49.2 years ( \pm 11.6 years). $25 \%$ of patients below $39,50 \%$ of patients below 50 and $75 \%$ of patients below 59.75 .
Figure (4) shows that out of 100 patients, there were 68 females representing $68 \%$ of the study population and 32 males representing $32 \%$ of the study population.

Figure (5) shows that out of 100 patients in our study: There were (21) patients were diabetic, (26) patients were hypertensive, (18) patients had chronic obstructive pulmonary disease and (5) patients had ischemic heart disease.

Figure (6) shows that out of 100 patients in our study, there were (35) patients had previous abdominal surgery and 65 patients had no previous abdominal surgery of our study.

Figure (7) shows that $60 \%$ of operations were carried out under spinal anesthesia and $40 \%$ of operations were carried out under general anesthesia.

Figure (8) shows that out of 100 patients of our study: in $28 \%$ of cases the hernial content was intestine and in 72 of cases the hernial content was omentum.

Figure (9) shows that out of 100 patients of our study, there were developing of significant seroma in (30\%) of patients as follow: (17\%) at group A (with drain) and (13\%) at group B (without drain). Significant seroma was not seen in $(70 \%)$ of patients.

Figure (10) shows that out of 100 patients of our study, the incidence of hematoma was (20\%) of patient as follow: (14\%) for group A (with drain) and (6\%) for group B (without drain), hematoma not seen in $(80 \%)$ of patients. Regarding follow up of hematoma cases, 17 cases resolved completely after adequate follow up and medical treatment, 2 cases developed infection that resolved after antibiotic therapy and only 1case developed early recurrence.

Figure (11) shows that out of 100 patients of our study: wound infection was developed in $(18 \%)$ of patients as follow; (12\%) for group A (with drain) and (6\%) for group B (without drain). wound infection not seen in $(82 \%)$ of patients. Regarding follow up of infection cases, 17 cases resolved with follow up and medical treatment, only one case developed early recurrence.

Figure (12) shows that out of 100 patients of our study: (2\%) of total count only showing early recurrence that present at group $\mathrm{B}$.

Figure (13) shows that out of 100 patients of our study: (9\%) of total count only complained of persistent postoperative pain as follows, 6 cases from group (A) and 3 cases from group (B).

Figure (14) shows that mean hospital stay was 1.46 days for group (A) and 1.26 days for group (B).

Figure (15) shows that mean period before returning to normal activity was 3.7 weeks for group (A) and 2.9 weeks for group (B). 
Table (1): Distribution of patients according to age per years

\begin{tabular}{|l|l|}
\hline Age & \\
\hline Mean & 49.2833 \\
\hline Std. Deviation & 11.67801 \\
\hline Minimum & 27.00 \\
\hline Maximum & 70.00 \\
\hline \multirow{3}{*}{ Percentiles } & 39.0000 \\
\hline & 50.0000 \\
\hline & 59.7500 \\
\hline
\end{tabular}

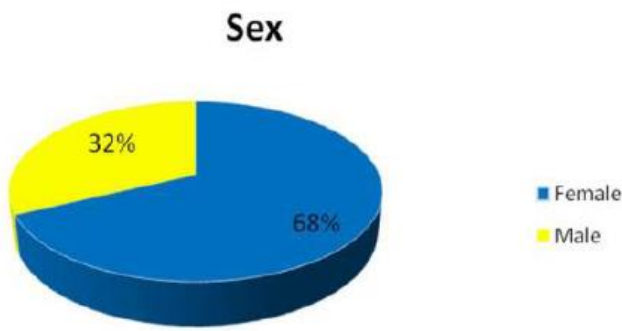

Figure (4): Sex

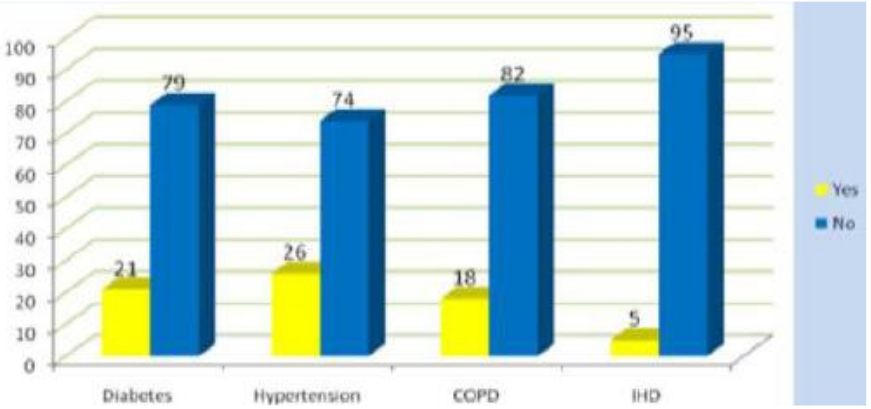

Figure (5): Distribution of patients according to medical history.

\section{Abdominal surgery}

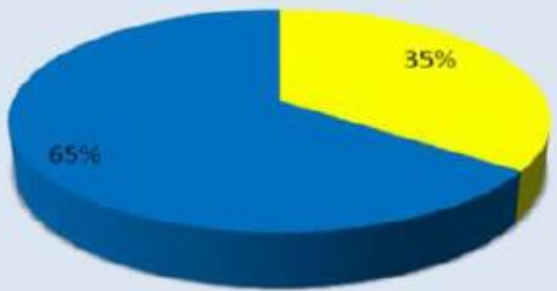

Figure (6): Abdominal surgery

\section{Anesthesia Type}

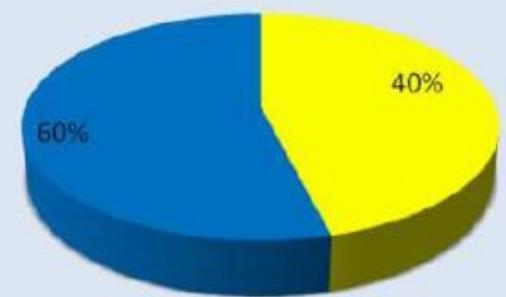

General

espina

Figure (7): Distribution of patients according to anesthesia type.

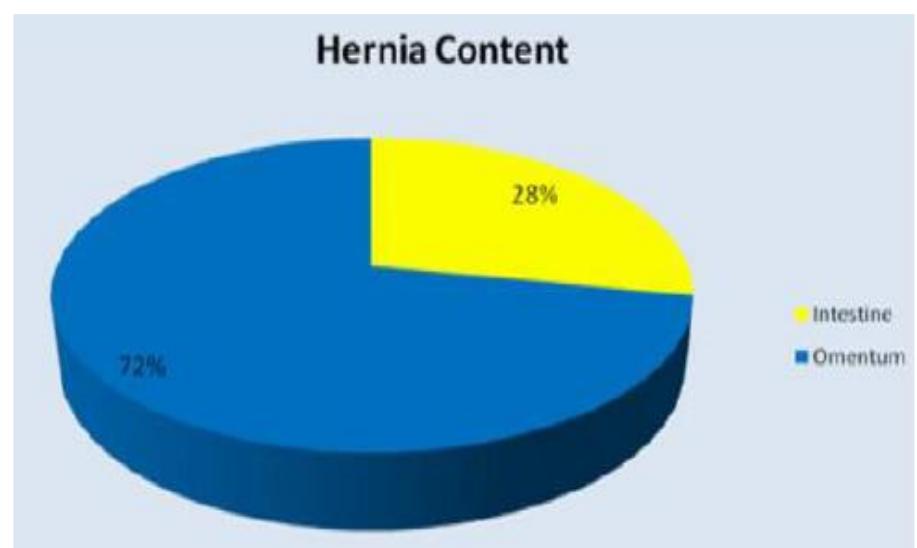

Figure (8): Distribution of patients according to hernia content

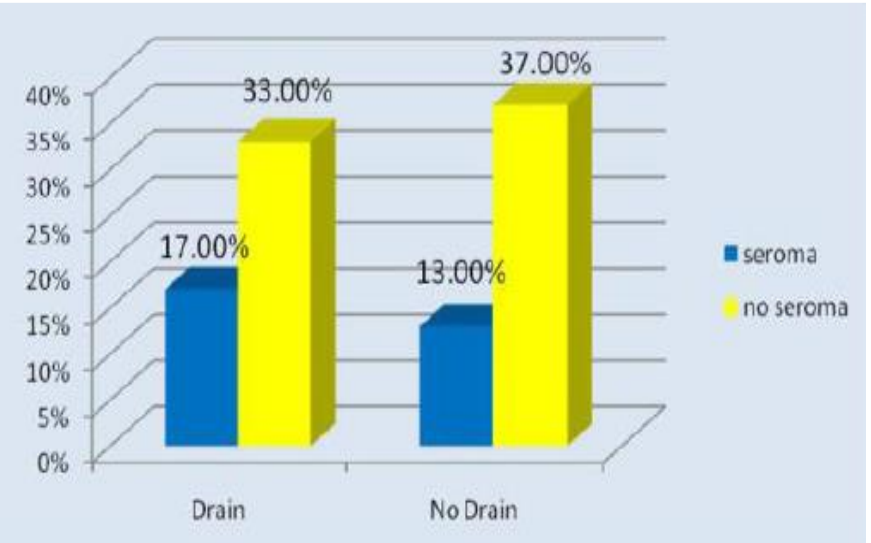

Figure (9): Significant seroma

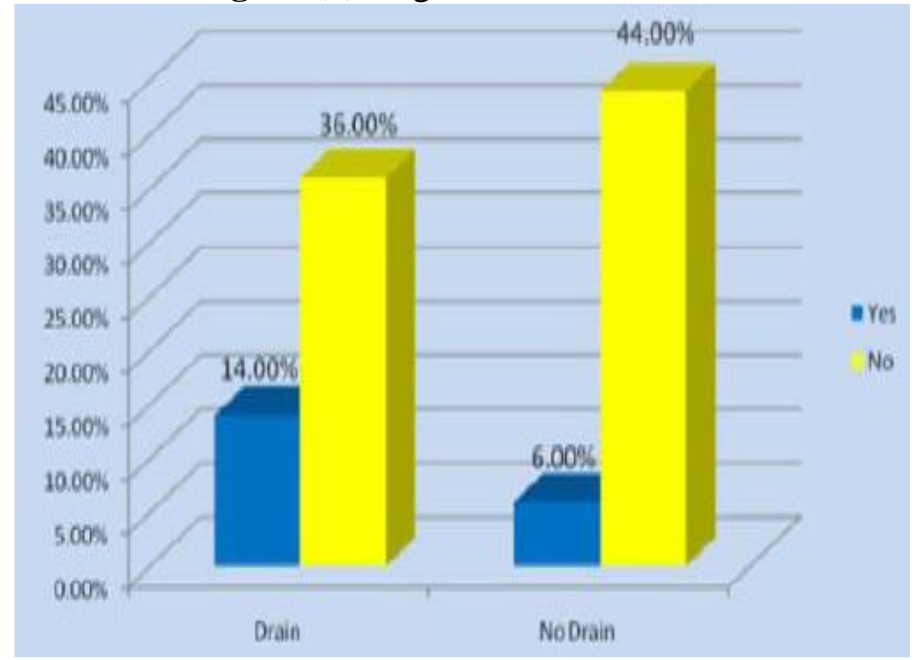

Figure (10): Hematoma 
Ahmed El-Tokhy

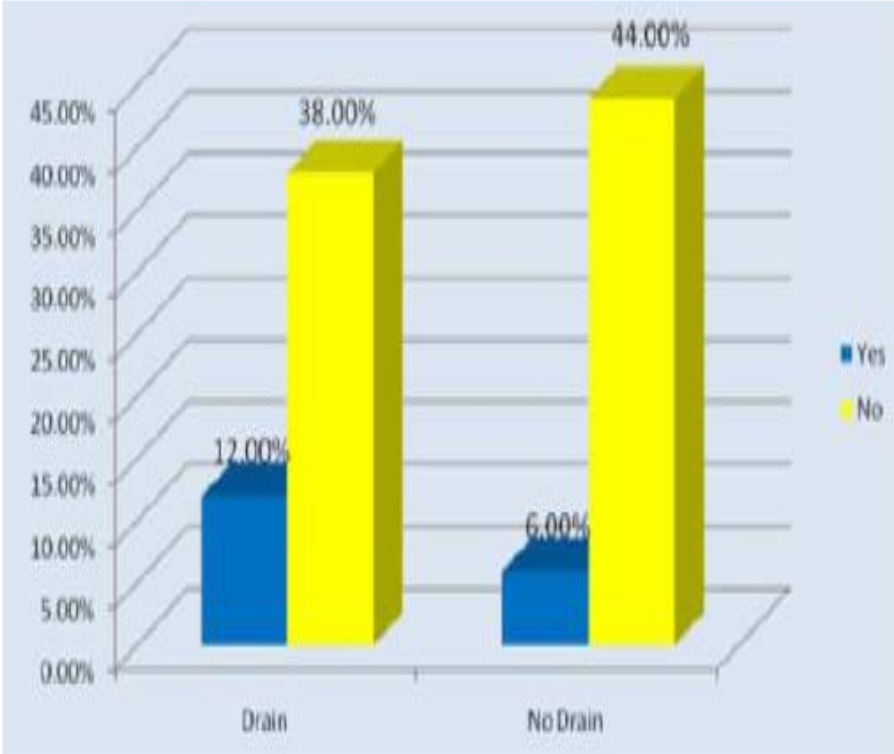

Figure (11): Wound Infection

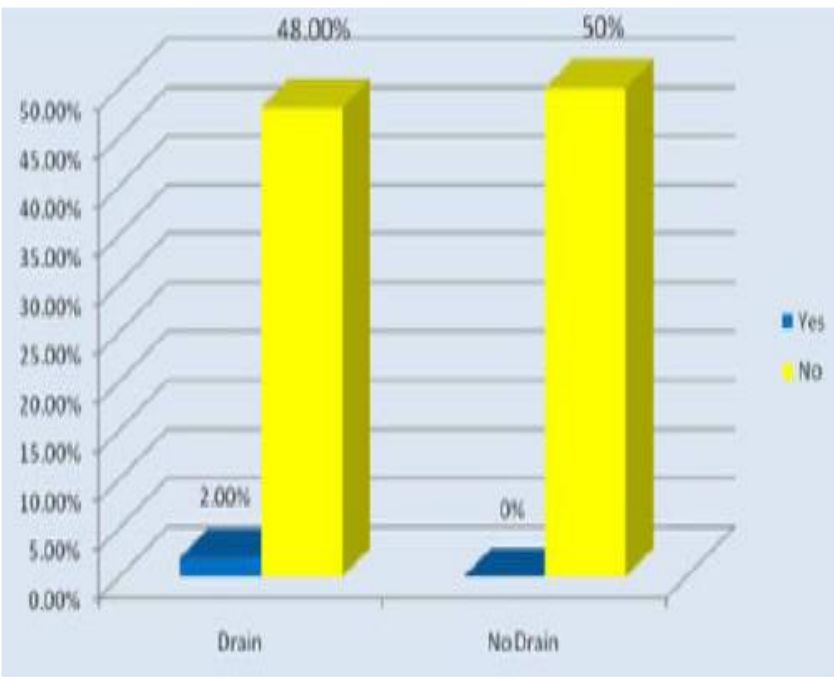

Figure (12): Early recurrence

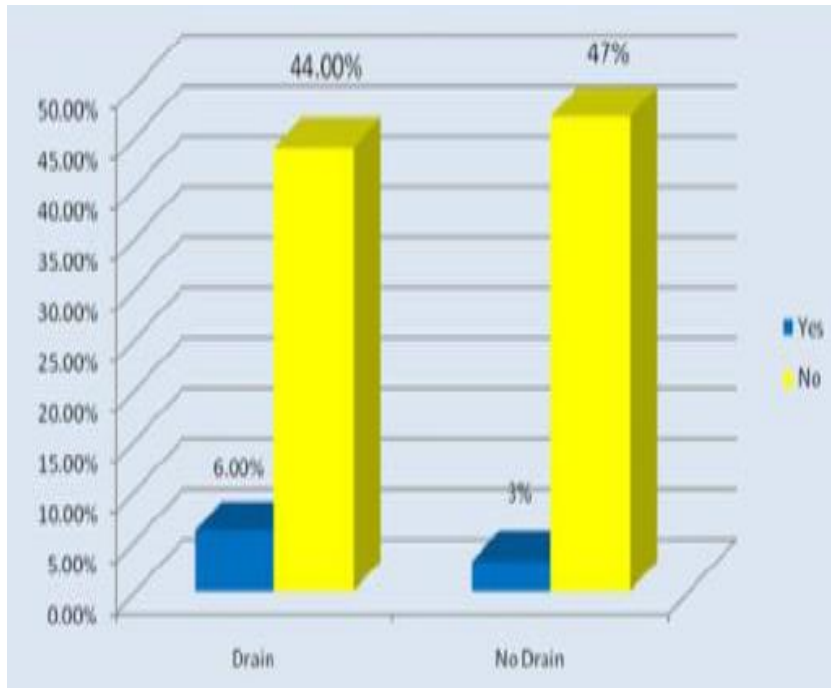

Figure (13): Persistent postoperative pain

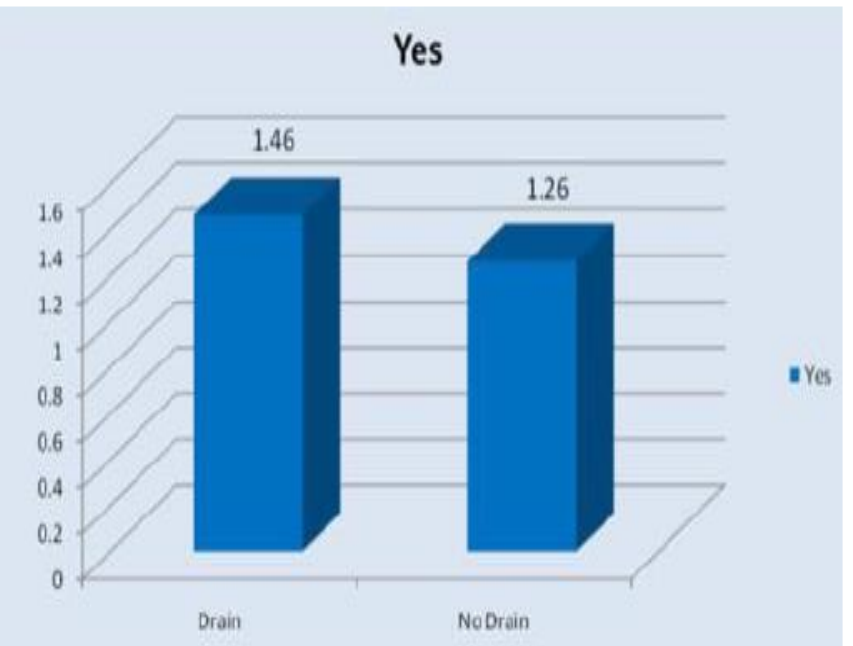

Figure (14): Postoperative hospital stay

\section{Yes}

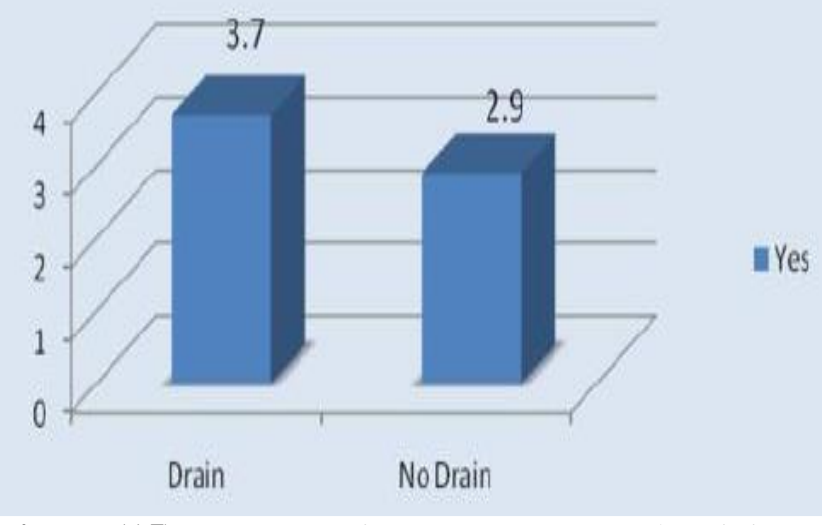

Figure (15): Postoperative return to normal activity 


\section{DISCUSSION}

Ventral hernias are commonly encountered in surgical practice. The estimated incidence of ventral hernia is $15-20 \%$. An abdominal incisional hernia represents a failure of primary laparotomy wound healing or a failure of a previous hernia repair, due to many local and general factors. The repair of ventral hernias has evolved from simple suture approximation to the use of prosthetic mesh and, recently, laparoscopic procedures ${ }^{(3)}$.

Hernias that are less likely to incarcerate include upper abdominal hernias, hernias less than $1 \mathrm{~cm}$ in diameter, and hernias larger than 7 to $8 \mathrm{~cm}$ (where loops of bowel can move in and out of the hernia sac without restriction, and are therefore less likely to become incarcerated) ${ }^{(4)}$.

Paraumbilical hernia is a protrusion through the linea alba just above or below the umbilicus, if the hernia is untreated it increase in size and more and more of its contents become irreducible eventually strangulation may occur thus operation should be advised in nearly all cases ${ }^{(4)}$.

As a result of high recurrence rate in the repair of ventral hernia, various types of repairs have been used both anatomical and prosthetic. But the results have been disappointing with a high incidence of recurrence of about 30-50\% after anatomical repair and $1.5-10 \%$ following prosthetic mesh repairs ${ }^{(4)}$.

Although drains are placed at the end of surgery as an attempt to prevent such complications, there is no concrete evidence demonstrating their actual benefits or whether they might actually increase the incidence of infection. Other techniques are also used to prevent seroma formation, including progressive tension sutures, which are recommended by some authors for abdominoplasty ${ }^{(5)}$.

A Cochrane systematic review on the prophylactic use of drains following incisional herniorrhaphy did not locate any study demonstrating their usefulness ${ }^{(6)}$.

In our study, a total of 100 patients with noncomplicated abdominal wall (ventral) hernia were enrolled to review and assess the difference between insertion of wound drain and the use of abdominal binder and percutaneous aspiration after open repair of ventral hernias and its effect on postoperative outcome. Patients were divided into two groups: Group (A) includes 50 patients with insertion of wound drain. Group (B) includes 50 patients with application of abdominal binder immediately postoperatively for 2 weeks.

This study showed distribution of patients according to age, ranging from 27 years to 70 years with mean age of 49.2 years ( \pm 11.6 years). $25 \%$ of patients below 39, 50\% of patients below 50 and $75 \%$ of patients below 59.75. Bhattaria and Bhandari (7) found a mean age of over 50 years which is some what more than the detected age in our group of patients.

The study revealed that out of 100 patients, there were 68 females (68\%) and 32 males (32\%).

Hameed et al. ${ }^{(8)}$ and Memon et al. ${ }^{\left({ }^{(9)}\right.}$ indicated that the female to male sex ratio was 1.4:1. The incidence of ventral hernia is higher in females rather than males but this difference was non-significant.

Clarke ${ }^{(10)}$ found no significance difference in both groups of his study as regards sex incidence. Bhattaria and Bhandari (7) found an increased incidence of incisional hernia in females $(72.3 \%)$ than males $(27.7 \%)$ in their study which agrees with our results.

In our study, $60 \%$ of operations were carried out under spinal anesthesia and $40 \%$ of operations were carried out under general anesthesia. Out of 100 patients of our study, in $28 \%$ of cases, the hernial content was intestine and in 72 of cases the hernial content was omentum.

Out of 100 patients of our study, there were developing of significant seroma in $(30 \%)$ of patients as follow: (17\%) at group A (with drain) and (13\%) at group B (without drain). Significant seroma was not seen in $(70 \%)$ of patients. In the results reported by Birolini et al. (11), the peak incidence of seroma formation occurs approximately two weeks after surgery, when prophylactic drains would be useless.

Westphalen et al. ${ }^{(12)}$ evaluated the occurrence of seroma and surgical wound infection after surgery. The incidence of postoperative seroma was $80 \%$ in recurrent hernias and $62.5 \%$ for non-recurrent hernias. There was no significant difference between the cases recurrent or nonrecurrent in the development of seroma $(\mathrm{p}=0.451)$.

Regarding hematoma, the incidence was $(20 \%)$ of patient as follow: (14\%) for group A (with drain) and (6\%) for group B (without drain), hematoma not seen in $(80 \%)$ of patients. Regarding follow up of hematoma cases, 17 cases resolved completely after adequate follow up and medical treatment, 2 cases developed infection that resolved after antibiotic therapy and only 1case developed early recurrence.

Wound infection was developed in (18\%) of patients as follow; (12\%) for group A (with drain) and (6\%) for group B (without drain). wound infection not seen in $(82 \%)$ of patients. Regarding follow up of infection cases, 17 cases resolved with follow up and medical treatment, only one case developed early recurrence. In the study by Memon et al. ${ }^{(13)}$, the incidence of infection among individuals was $21.67 \%$. Westphalen et al. (12) found that patients with recurrent hernias had a $30 \%$ postoperative infection rate against $18.75 \%$ in the non-recurrent. There was no significant difference between the cases recurrent or nonrecurrent in the development of postoperative infection $(\mathrm{p}=0.660)$. 
In our study, out of 100 patients of our study, (2\%) of total count only showing early recurrence that present at group B. (9\%) of total count only complained of persistent postoperative pain as follows, 6 cases from group (A) and 3 cases from group (B). Mean hospital stay was 1.46 days for group and 1.26 days for group (B). Mean period before returning to normal activity was 3.7 weeks for group (A) and 2.9 weeks for group (B).

Canziani et al. (14) recorded post-operative wound infection in $10 \%$ and wound hematoma in $7.5 \%$ which is in agreement of our study but did not record seroma formation which is in contradiction of our results. They recorded a post-operative hospital stay of 2-8 days with a mean of 3 days. Also, Bhattaria and Bhandari (7) found post-operative hospital stay of about 3 days mean in their study group.

Potential complications of incisional hernia surgery include hernia recurrence, infection, seromas, hematomas, adhesions, bowel obstruction and erosion of mesh into the bowel or urinary bladder ${ }^{(\mathbf{1 5})}$.

Clarke ${ }^{(10)}$ found no significance difference between the two groups of his study as regards postoperative complications (chronic pain, recurrence and wound infection).

Westphalen et al. ${ }^{(12)}$ stated that there was no loss of follow-up and all cases were analysed. No participant died or exhibited recurrence of hernia along the 30-day postoperative follow-up. They concluded that the frequency of seroma and infection did not exhibit significant differences between individuals subjected to only mesh repair of large incisional hernias with drains or progressive tension sutures without drainage.

\section{CONCLUSION}

It could be concluded that combined abdominal binder and percutaneous aspiration for selected cases were superior to insertion of wound drains as regards seroma formation, wound infection and postoperative return to normal activity with no significant difference as regards hematoma, postoperative pain, hospital stay and patient satisfaction.

\section{REFERENCES}

1. Helgstrand F, Rosenberg J, Bay-Nielsen $M$ et al. (2010): Establishment and initial experiences from the Danish ventral hernia database. Hernia, 14(2):131-135.

2. Alnassar S, Bawahab M, Abdoh A et al. (2012): Incisional hernia postrepair of abdominal aortic occlusive and aneurysmal disease: Five-year incidence. Vascular, 20(5):273-277.

3. Barbaros U, Demirel T, Sumer A et al. (2011): Incisional hernia repair. J Instit Med., 32(1): 34-37.

4. George, CD, Ellis H (2010): The results of incisional hernia repair: a twelve year George $\mathrm{CD}$, Ellis $\mathrm{H}$. The results of Incisional hernia repair. A twelve year review. Ann R Coll., 68:185-188.

5. Pollock TA, Pollock H (2012): Progressive tension sutures in abdominoplasty: a review of 597 consecutive cases. Aesthet Surg J., 32(6):729-42.

6. Gurusamy KS, Allen VB and Samraj K (2012): Wound drains after incisional hernia repair. Cochrane Database Syst Rev., 2: CD005570.

7. Bhattaria A, Bhandari RS (2010): Incisional hernia repair. J Instit Med., 32(1): 34-37.

8. Hameed F, Ahmed B, Ahmed A et al. (2009): Incisional hernia repair by preperitoneal (sublay) mesh implantation. APMC .,3(1): 27-31.

9. Memon MR, Shaikh AA, Memon SR et al. (2010): Results of stoppa's sublay mesh repair in incisional and ventral hernias. JPMA., 60:798.

10. Clarke MH (2010): Incisional hernia repair by fascial component separation: results in 128 cases and evolution of technique. Am J Surg., 200: 2-8.

11. irolini C, de Miranda JS, Utiyama EM et al. (2015): A retrospective review and observations over a 16-year clinical experience on the surgical treatment of chronic mesh infection. What about replacing a synthetic mesh on the infected surgical field? Hernia, 19(2): 239-46.

12. Westphalen AP, Araujo ACF, Zacharias $P$ et al. (2015): Repair of large incisional hernias: To drain or not to drain. Randomized clinical trial. Acta Cirurgica Brasileira, 30(12): 844-851.

13. Memon AA, Khan A, Zafar H et al. (2013): Repair of large and giant incisional hernia with onlay mesh: perspective of a tertiary care hospital of a developing country. Int J Surg., 11(1): 41-5.

14. Canziani M, Frattini F, Cavalli M et al. (2009): Sutureless mesh fibrin glue incisional hernia repair. Hernia, 13: 625-629.

15. Gurusamy KS, Samraj K (2010): Wound drains after incisional hernia repair. Cochrane Database Syst Rev., 6:

CD005570 